\title{
Flexible job shop scheduling strategy with uncertain processing time
}

\author{
Bo Bao 1, a , Tifang $\mathrm{Li}^{1,}$, , Bo Zhang ${ }^{1, \mathrm{c}}$ \\ Air Force Engineering University, Xi'an Shanxi 710051, China \\ a18220553171@163.com, b962400117@qq.com, c1114573871@qq.com
}

\begin{abstract}
Keywords: uncertain processing time, Flexible job shop scheduling, distinct due window.
\end{abstract}
\begin{abstract}
The processing time is one of the most common uncertain factors in the workshop production, which has a significant influence on the scheduling of production. In order to address the flexible job shop scheduling problem with uncertain processing time, a flexible job shop scheduling combined strategy is proposed. The fuzzy scheduling model with time windows was established based on fuzzy programming theory, which is then transformed into distinct scheduling model by using the algorithm of maximizing the membership function of middle value, two rescheduling methods corresponding to tardiness disturbance was proposed, and the genetic algorithm was used for optimization. The feasibility and effectiveness of the scheduling method and algorithm are verified by simulation experiment.
\end{abstract}

\section{Introduction}

In production scheduling, due to operating workers skill differences, process route change, job repair and other reasons, processing time is uncertain, and it is a class of most common uncertain factors in production scheduling. At present, there are three kinds of methods to describe the uncertain factors, such as interval number [1, 2], fuzzy number [3, 4] and random number [5, 6]. Among them, the fuzzy number can effectively use the expert experience and historical data, which showing the good practicality and applicability. Fardin Ahmadizar [7] used a triangular fuzzy number method to deals with a fuzzy group shop scheduling problem. Chia-Yu Hsu [8] proposed a negotiation mechanism based on fuzzy constraints, solve the distributed job shop scheduling problem effectively. For the fuzzy flexible job shop scheduling problem, Deming Lei [9] proposed a co-evolutionary genetic algorithm, Jun-qing $\mathrm{Li}$ [10] proposed a chemical-reaction optimization algorithm for flexible job-shop scheduling problems with maintenance activity. For the study, fuzzy scheduling model effectively utilizes the ability of fuzzy number to deal with the uncertainty of variables, but there is a problem that the scheduling scheme generated are fuzzy numbers, and when the quantity of scheduling operations is large, the fuzzy features makes the guidance of scheduling scheme decreased. $\mathrm{Yu}$ Shengping [11] used the method that transform the fuzzy scheduling model into a distinct nonlinear programming model, solving the problem of the uncertainty processing time in the production, but the problem is didn't considered the method to deal with the disturbance.

In this paper, aiming at the problem of the flexible job shop scheduling with uncertain processing time, we established the triangular fuzzy number fuzzy scheduling model, and transform the fuzzy scheduling model into a distinct scheduling model by using the algorithm of maximizing the membership function of middle value, put forward the corresponding tardiness disturbance response strategy, and used the genetic algorithm for optimization.

The remainder of the paper is organized as follows. The triangular fuzzy number scheduling model is introduced in Section 2. The combined scheduling strategy of flexible job shop scheduling with uncertain processing time is described in Section 3. The proposed GA is described in Section 4, and Section 5 provides the experimental results. The paper is concluded in the final section. 


\section{Problem description and analysis}

\subsection{Triangular fuzzy number operation definition.}

In this paper, the triangular fuzzy number is used to describe the uncertain parameters. The triangular fuzzy number is denoted as $\tilde{r}$, as $\left(r^{L}, r^{M}, r^{U}\right)$, and the membership function is:

$$
\mu(r)=\left\{\begin{array}{cl}
\frac{r-r^{L}}{r^{M}-r^{L}} & r^{L} \leq r \leq r^{M} \\
\frac{r^{U}-r}{r^{L}-r^{M}} & r^{M} \leq r \leq r^{U} \\
0 & \text { others }
\end{array}\right.
$$

In this paper, two kinds of fuzzy rules are used. Among them, the addition operation of two fuzzy numbers $\tilde{r}=\left(r^{L}, r^{M}, r^{U}\right), \tilde{t}=\left(t^{L}, t^{M}, t^{U}\right)$ is defined as:

$$
\tilde{r}+\tilde{t}=\left(r^{L}+t^{L}, r^{M}+t^{M}, r^{U}+t^{U}\right)
$$

Maximum operation:

$$
\max \{\tilde{r}, \tilde{t}\}=\left(r^{L} \vee t^{L}, r^{M} \vee t^{M}, r^{U} \vee t^{U}\right)
$$

\subsection{Triangular fuzzy number model.}

In this paper, the flexible job shop scheduling problem is studied under the condition that the operation is uncertain and the distinct due window. The completion time of the work is out of the due window, whether it is earliness or tardiness, are faced with earliness / tardiness penalties.

The scheduling problem can be described as: $n$ jobs are processed in the $m$ machines, $i=1,2, \cdots, n, j=1,2, \cdots, m$. $O_{i k}$ Is the $k$ th operation of job. Job can choose machine $M_{j}$ to carry out the operation $O_{i k}$, expressed as $p_{i j k}$. $T_{i j k}$ Is the corresponding maintenance time of $p_{i j k}$. Jobs are in accordance with the processing order, and each operation can processed on some machines. The purpose of the optimization of the scheduling problem is to make the scheduling scheme to achieve the optimal performance index by arrange the operations of the job on the machine scientifically, under the relevant constraints.

Based on the above analysis, the problem can be described as a mathematical model as :

$$
\begin{aligned}
& G=\sum_{i=1}^{n}\left(h_{i} \max \left(0, e_{i}-\tilde{c}_{i}\right)+w_{i} \max \left(0, \tilde{c}_{i}-t_{i}\right)\right) \\
& \quad \sum_{l}^{m} x_{i j k}=1, \forall i, \forall j, \forall k, \\
& \text { s.t. } \\
& \tilde{e}_{i k}=\tilde{s}_{i k}+T_{i j k} \\
& \tilde{e}_{i k} \leq \tilde{s}_{i(k+1)} \\
& \tilde{e}_{j u} \leq \tilde{s}_{j(u+1)} \\
& \tilde{c}_{i}=\tilde{e}_{i l}
\end{aligned}
$$

Formula (4) is the objective function, the purpose is to minimize the earliness, tardiness penalties value, also a triangular fuzzy number, expressed as $G=\left(G^{L}, G^{M}, G^{U}\right) .\left[e_{i}, t_{i}\right]$ is the due window of job $i$. $h_{i}, w_{i}$ are the weight of earliness, tardiness penalties. $\tilde{c}_{i}$ is the completion time of job $i$. Formula (5) described that each operation can chose only one machine, $x_{i j k}$ is $0 / 1$ variables, described as operation $O_{i k}$ whether choose the machine $M_{j}$. Formula (6) indicates the processing time parameter constraint. Formula (7), (8) indicates the time constraints of operation of job, and the time constraints of operations on each machine, and the $s_{i u}, e_{i u}$ are the start and end time of the $u$ th operation on machine $M_{i}$. Formula (9) indicates the completion time of each job. 


\subsection{Problem analysis.}

Using the triangular fuzzy number to describe the uncertain processing time can use the actual information effectively. However, by using the fuzzy number model, the start, and end time of the scheduling scheme are fuzzy number. Moreover, with the increase of the number of operations, the fuzziness can appear the "cumulative effect ".

Figure 1 is a Gantt chart of a fuzzy scheduling result, in the figure, the triangle below the line denote the start time of operation, the triangle above the line denote the end time of operation, the figures in brackets denote the number of job and operation. It can be intuitively understood that with the increase of the quantity of operations, the scope of the start / end time of the subsequent operation is enlarged, which leads to the difficulty of the scheduling scheme to effectively guide the production work.

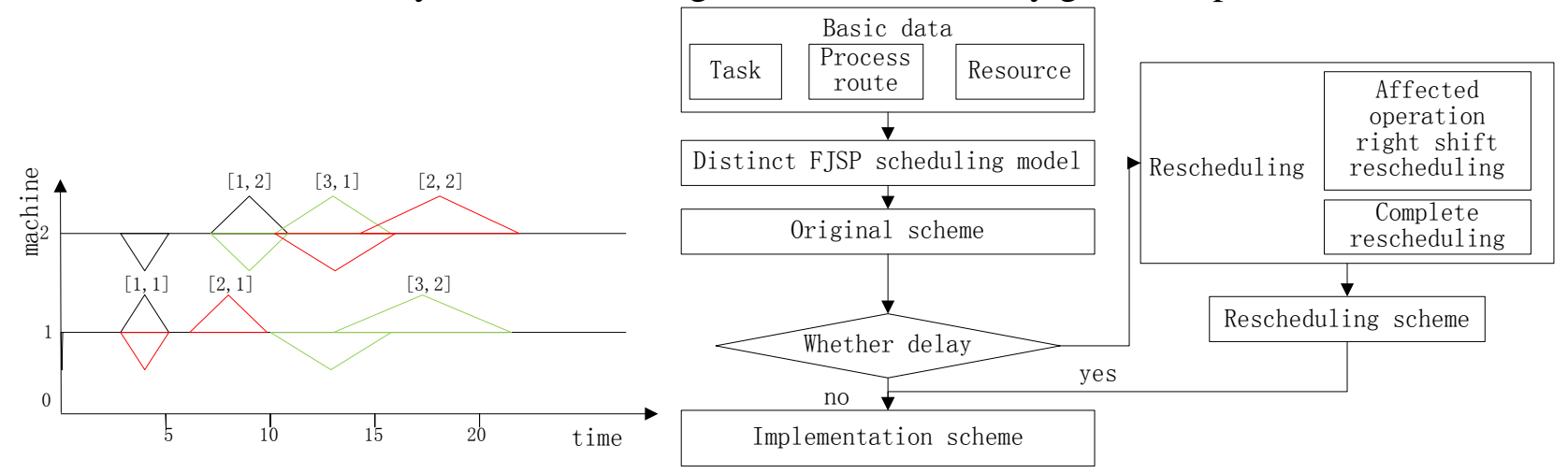

Fig. 1 Scheduling scheme Gantt chart

Fig. 2 Flow of scheduling method

\section{Flexible job shop scheduling combined strategy}

According to the above analysis, the model is transformed into a distinct scheduling scheme based on the triangular fuzzy number model, and the rescheduling methods corresponding to tardiness disturbance is proposed.

\subsection{Operation flow.}

Firstly, according to the task data, process route and other basic information, establish the triangular fuzzy number scheduling model. Then, transform the fuzzy programming model into distinct programming model based by using the algorithm of maximizing the membership function of middle value, and form the initial scheduling scheme. If the disturbance occurs, on the basis of disturbance time, use the rescheduling method to adjust the scheme. Among them, the key technologies include the method of model transformation and the design of rescheduling strategy.

The flow of the scheduling method is shown in the figure as Figure 2.

\section{2 model transformation.}

The fuzzy programming model is transformed into a distinct planning model, so that a distinct scheduling scheme can be obtained. the intermediate value maximum membership degree algorithm is used to transform the fuzzy programming model into a distinct single objective nonlinear programming model. The model is described as follows:

$$
\begin{aligned}
& \max \left\{\Gamma \alpha^{L}+(1-\Gamma) \alpha^{U}\right\} \\
& \text { s.t. } \alpha^{U} \geq \mu_{i} \geq \alpha^{L} \quad i=1,3 \\
& \mu_{2} \geq \alpha^{U} \\
& \alpha^{L}, \alpha^{U} \in[0,1]
\end{aligned}
$$

Set $G^{L}, G^{M}, G^{U}$ which in the orgin model as the most pessimistic, the most likely and the most optimistic value. Among them, the operator $\Gamma$ reflects the degree of attention of decision makers for the most pessimistic, the most likely and the most optimistic value, given by the decision maker. Optimize the three object functions at one time and form a distinct multi-objective linear programming model, which can be determine three satisfaction membership functions $\mu_{i}(i=1,2,3) . \alpha^{L}$ is the 
minimum value of $\mu_{i}, \alpha^{L}$ the maximum value of $\mu_{i}$. Finally, model optimizing with the objective function $\max \left\{\Gamma \alpha^{L}+(1-\Gamma) \alpha^{U}\right\}$, and get a distinct scheduling scheme.

\subsection{Scheduling methods.}

In the scheduling process, check the end time node of scheme of each operation, estimate whether there is a delay, and estimate the tardiness quantity. Considering the two factors of both the scheduling performance index and the resources consumption of reallocation, and select the appropriate rescheduling method under the condition of different disturbance degree.

\subsubsection{Offset calculation.}

The main purpose of rescheduling is to reduce the influence of the tardiness of the operation, and to meet the requirements of satisfaction. However, considering the actual situation, the change of the plan will bring the cost of adjustment of machine and resources. Thought set the threshold, to find a balance point between the rescheduling cost and scheduling satisfaction. By comparing the offset of the disturbance degree with the threshold value, the paper provides the basis for the selection of rescheduling methods. Set the threshold value as $\theta_{0}$, the offset as $\theta$, as follows:

$$
\theta=\lambda_{1} \sum_{i=1}^{n}\left|w_{i} \frac{c_{i}{ }^{\prime}-c_{i}}{c_{i}}\right|+\lambda_{2} \sum_{i=1}^{n} \sum_{l=1}^{k}\left|\frac{e_{i k}{ }^{\prime}-e_{i k}}{e_{k}}\right|
$$

Where the first half part describes the degree of deviation of job completion time, reflects the requirement of satisfaction, $c_{i}^{\prime}, c_{i}$ denote the completion time of scheduling scheme of tardiness and the completion time of origin scheme. The second part describes the deviation degree of the operation of the job, reflects the cost for scheme adjustment, $t_{i l}{ }^{\prime}, t_{i l}$ denote the operation end time of scheduling scheme of tardiness and the operation end time of origin scheme, $\lambda_{1}, \lambda_{2}$ denote the punishment coefficient of this two index. Among them, the scheduling scheme of tardiness is obtained by the affected operation right shift rescheduling method.

\subsubsection{Affected operation right shift rescheduling.}

Affected operation right shift rescheduling method implement the reactive scheduling only for the direct or indirect operation affected by the tardiness. When $\theta \leq \theta_{0}$, as the offset is less than the threshold value, adopting the affected operations right shift rescheduling method. Analyzing the impact operation, described as the form of operation related tree, set the tardiness operation as the root node, set the following operation on job and following operation on machine as the first order son nodes, and set multi order affected son nodes step by layer by layer, until there is no more following operation.

The specific steps of affected operation right shift rescheduling method are as follows:

Step 1: Positioning the tardiness operation, and built the operation related tree by the way of binary tree structure.

Step 2: Adjust the delay operation start/end time, and set the tardiness operation as the parent node.

Step 3: Put the end time of parent node in new scheme as the start time of the following operation on job and the following operation on machine, reassignment and adjustment, and put then as the new parent node.

Step 4: If the parent node does not contain a child node, turn to Step 5, otherwise go to Step 3.

Step 5: End of dynamic adjustment.

\subsubsection{Complete rescheduling.}

The complete rescheduling method is to rescheduling all the operations that have not been processed, and generate a new scheduling scheme when the disturbance occurs. When $\theta \geq \theta_{0}$, the offset is greater than the threshold, adopt the complete rescheduling method.

An important difference between complete rescheduling and static scheduling is that the processing start time of each machine is different. The complete rescheduling needs to give a rescheduling start time firstly, and delete the unstated operations in that moment, and back to unadjusted state. In addition, due to the scheduling is not every operation, but some parts of operations, so it is necessary to establish a set of scheduling operations, and rescheduling based on the operations set. 


\section{Algorithm}

The problem of flexible job shop scheduling is a typical NP hard problem and the uncertainty of operations time increases its complexity. The genetic algorithm shows great applicability in solving similar problems. Therefore, this paper used the genetic algorithm to solve the scheduling problem so as to form an initial schedule. Additionally, rescheduling the scheme based on the initial schedule when disturbances occur.

\subsection{Chromosome coding.}

The chromosome is encoded in integer, and each chromosome represents a feasible solution to the problem. The chromosome an integer string which the length is $2 \sum_{i=1}^{n} D_{i} m_{i}, m_{i}$ is denoted the operations quantity of job $D_{i}$, The first half of the chromosome represents the sequence of processing of all the jobs on the machine, and the latter indicates the corresponding equipment of each operation. For example:

$[2,1,1,2,3,3,1,2,3,1,2,3,3,1,2,3,2,1]$

Without considering the specific value of the operation time, the chromosome can be decoded and represented as a Gantt chart as shown in Figure 3, and the " $a-b "$ in figure represents the $b$ th operation of job $a$.

\begin{tabular}{|c|c|c|c|c|}
\hline$\Xi 3$ & & $1-2$ & $2-2$ & $1-3$ \\
\hline 2 & $1-1$ & & $3-2$ & $2-3$ \\
\hline$\stackrel{\pi}{2} 1$ & $2-1$ & $3-1$ & & $3-3$ \\
\hline
\end{tabular}

\subsection{Fitness calculation.}

Fig. 3 Scheduling scheme Gantt chart

The objective function of the transformed scheduling model is used as fitness function, and the fitness value of the chromosome is calculated baced on it, the calculating formula:

$$
\text { fitness }=\max \left\{\Gamma \alpha^{L}+(1-\Gamma) \alpha^{U}\right\}
$$

The greater the fitness value, the better the corresponding chromosome.

\subsection{Select operation.}

According to the fitness value of chromosome selection, this paper uses the roulette wheel selection method, the better the greater value, the greater the probability of selection:

$$
p(i)=\text { fitness }(i) / \sum_{i=1}^{n} \text { fitness }(i)
$$

\subsection{Crossover operation.}

Select two chromsomes from the population of chromsomes, for the first half of the chromosome, select the cross location randomly and take the cross treatment. Cross operation such as: assume the cross position is 3 , and takes the cross operation for following two chromosomes for the first 3 positions.

$$
\begin{aligned}
& \text { chrosome 1:[2,1,3,1,2,3,1,2,3,3,2,1] } \text { crossover }_{\rightarrow}[3,3,1,1,2,3,1,2,3,3,2,1] \\
& \text { chrosome } 2:[3,3,1,2,1,2,1,2,3,1,2,3] \rightarrow[2,1,3,2,1,2,1,2,3,1,2,3]
\end{aligned}
$$

After the cross operation, some operation will excess or lack, in this regard, turn the excess operations into a lack operation, and the second half of the chromosome unchanged, thus avoiding the generation of illegal chromosomes. For example, the adjustment of individual 1 is as follows:

$$
[3,3,1,1,2,3,1,2,3,3,2,1] \stackrel{\text { adjust }}{\rightarrow}[3,3,1,1,2,2,1,2,3,3,2,1]
$$

\subsection{Mutation operation.}

New individuals were obtained by mutation to increase population diversity. The mutation selects a chromosome randomly, and selects two random points in the first half of the chromosome to exchange the jobs and the corresponding machines. For example, the selection of a chromosome in position 2, 3 cross mutation operation: 


\subsection{Algorithm implementation process.}

The specific operation of the algorithm is as follows:

Step1: Set algorithm parameters, and randomly generated initial population.

Step2: Calculate the fitness value of all the chromosomes.

Step3: Selection, crossover and mutation operations to form a new generation of population.

Step4: Termination judgment. Determine whether the number of iterations meet the termination conditions, if the conditions are met, output the optimal solution. Otherwise, return to Step2, take the cycle operation.

\section{Experiment and analysis}

Assuming that the factory uses 5 sets of equipment to process 4 jobs and each job has 4 operations. The optional processing machines and the corresponding fuzzy processing time as in table ([number of optional machine]/[ corresponding processing time(day)]), all the due windows are [25,30](day). The scheduling scheme is formed basing on the above information.

Table 1. Optional number of machine and corresponding time

\begin{tabular}{ccccc}
\hline jobs & $\mathrm{O}_{\mathrm{i} 1}$ & $\mathrm{O}_{\mathrm{i} 2}$ & $\mathrm{O}_{\mathrm{i} 3}$ & $\mathrm{O}_{\mathrm{i} 4}$ \\
\hline $\mathrm{D}_{1}$ & {$[1] /[(3,4,5)]$} & {$[2,3] /[(7,8,9),(8,9,10)]$} & {$[4] /[(7,9,11)]$} & {$[1,2] /[(3,4,5),(4,5,6)]$} \\
$\mathrm{D}_{2}$ & {$[1,2] /[(4,5,6),(5,6,7)]$} & {$[2,5] /[(6,8,10),(5,6,7)]$} & {$[3] /[(4,5,6)]$} & {$[1,5] /[(4,5,6),(5,6,7)]$} \\
$\mathrm{D}_{3}$ & {$[3] /[(5,6,7)]$} & {$[2,5] /[(4,5,6),(6,7,8)]$} & {$[4] /[[6,7,8]$} & {$[1,2] /[(4,5,6),(4,5,6)]$} \\
$\mathrm{D}_{4}$ & {$[5] /[(5,7,9)]$} & {$[2,3] /[(5,6,7),(4,5,6)]$} & {$[1,4] /[(4,5,6),(5,6,7)]$} & {$[1] /[(4,5,6)]$} \\
$\mathrm{D}_{5}$ & {$[2,3] /[(5,6,7),(3,4,5)]$} & {$[1,5] /[(4,5,6),(5,6,7)]$} & {$[2,3] /[(6,7,8),(8,9,10)]$} & {$[5] /[(7,8,9)]$} \\
\hline
\end{tabular}

\subsection{Parameter setting and generation scheme.}

The number of population was 40 , the maximum genetic algebra is 100 , the crossover rate is 0.8 , and the mutation rate is 0.6 . The operator $\Gamma$ is $0.5, h_{i}, w_{i}$ are 0.5 . Through the optimization, we get a distinct scheduling scheme, which is represented as Gantt chart, as shown in figure 4.

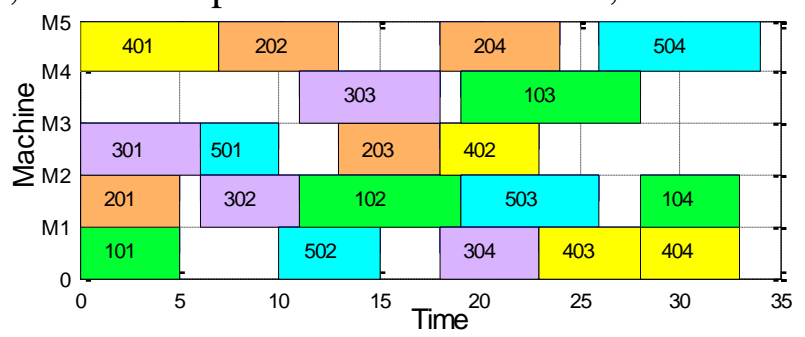

Fig. 4 Initial scheduling scheme Gantt chart

Through the genetic algorithm to solve the calculation, can give a set of distinct scheduling scheme. Compared with the fuzzy scheduling scheme, the explicit scheme is more conducive to the operator to understand the schedule, and is conducive to prepare the equipment, resources.

\subsection{Scheduling results analysis.}

When the tardiness disturbance occurs in the operation of implementing, it is necessary to deal with the rescheduling timely. The following experiments were carried out to analyze the effectiveness of the rescheduling method under two conditions, within the scope of the threshold value and exceed the scope of threshold value.

\subsubsection{Affected operation right shift rescheduling.}

Assuming that at the end of the operation $O_{23}$, that is, the 18th days of processing as the scheme in section 5.1, the tardiness disturbance occurs, and the experts predict the tardiness time is 2 days. By calculating and judging, the offset is less than the threshold value, so the rescheduling is carried out by the method of the affected operations right shift rescheduling. Use the GA to optimization, and the results shown in figure 5 . 


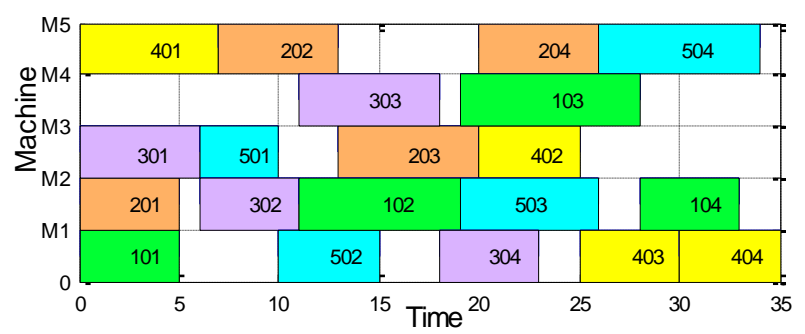

Fig. 5 Affected operation right shift rescheduling Gantt chart

It can be seen from the figure that the job following operation of $O_{23}$ is $O_{24}$, and the machine following operation of $O_{23}$ is $O_{42}$. When the tardiness disturbance occurs in the operation $O_{23}$, those operations will be impacted firstly, and then, the subsequent operations will be impacted, including the following operations of $O_{24}$ and $O_{42}$. In addition, the results show that the scheduling scheme doesn't change the selection of machines, and doesn't adjust every subsequent operation, which is conducive to maintain the scheme stability.

\subsubsection{Full rescheduling.}

Assuming that at the end of the operation $O_{23}$, that is, the 18th days of processing as the scheme in section 5.1, the tardiness disturbance occurs, and the experts predict the tardiness time is 10 days. By calculating and judging, the offset is greater than the threshold value, so the rescheduling is carried out by the method of the full rescheduling, and the results shown in figure 5 .

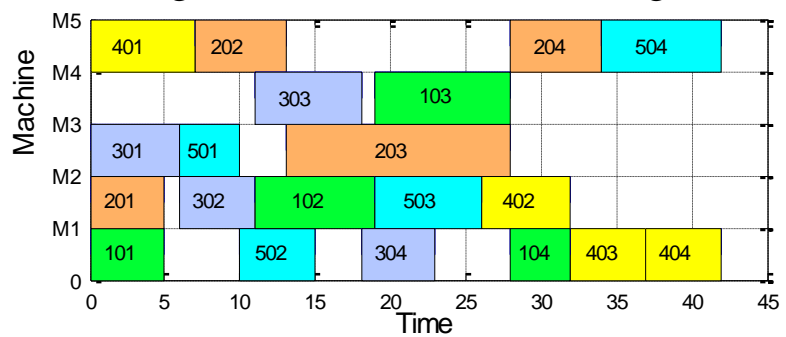

Figure 6 Full rescheduling Gantt chart

It can be seen from the figure that the tardiness disturbance occurs at the end of the operation $O_{23}$, predict that the tardiness time is long, and carried out by the method of the full rescheduling can effectively reduce the impact of the tardiness disturbance. If still use the affected operation right shift rescheduling method at this time, the job due to the order of the equipment, the order of the operation, the tardiness disturbance operation will have a greater impact on the subsequent operation. The rearrangement of the equipment and the order can effectively reduce the influence of the tardiness.

\section{Conclusion}

In conclusion, the flexible job shop scheduling problem with uncertain working hours is studied in this paper. Specifically, this paper analyzes the overall scheduling operation, establishes the flexible job shop scheduling model, and puts forward the combined rescheduling strategy to deal with the disturbance. The scheduling method can be used to form a distinct scheduling scheme, which is helpful for the operator to understand the accurate schedule, and is helpful for the effective allocation of equipment and resources. The rationality and effectiveness of the scheduling model and the method are verified by simulation experiments and results analysis. In the future research, it is necessary to further improve the accuracy of the initial program formulation and further design a stable and effective rescheduling strategy.

\section{References}

[1]. Yang Hongan, Xi Zhicheng, Xia Changkai,et,al. Minimax model and Two Space Co-evolutionary Genetic Algorithm for Job Shop Scheduling Problem[J]. China Mechanical Engineering, 2015, 26(3): 330-338. 
[2]. YANG Hong-an, WANG Zhou-feng, LYU Yang-yang, et al. Interval number solving method for job-shop scheduling problem with processing time variability [J]. Computer Integrated Manufacturing Systems, 2014, 20(9): 2231-2240.

[3]. Demion Lei. Scheduling fuzzy job shop with preventive maintenance through swarm-based neighbourhood search [J]. Int J Adv Manuf Technol, 2011, 54: 1121-1128.

[4]. Juan José Palacios, Jorge Puente, Camino R. Vela, ET, al. Benchmarks for fuzzy job shop problems [J]. Information Sciences, 2016:736-752.

[5]. GU J, GU M, CAO C, et al. A novel competitive co-evolutionary quantum genetic algorithm for stochastic Job-shop scheduling problem [J].Computer\& Operations Research, 2010, 37(5): 927-937.

[6]. AZADEH A, NEUAHBAN A, MOUHADDAM M. A hybrid computer simulation-artificial neural network algorithm for optimisation of dispatching rule selection in stochastic Job-Shop scheduling problems [J]. International Journal of Production Research.2012, 50(2):551-556.

[7]. Fardin Ahmadizar, Alireza Zarei. Minimizing make span in a group shop with fuzzy release dates and processing times [J]. Int J Adv Manuf Technol, 2013 66: 2063-2074.

[8]. Chia-Yu Hsu, Bo-Ruei Kao, Van Lam Ho, K. Robert Lai. Agent-based fuzzy constraint-directed negotiation mechanism for distributed job shop scheduling. [J]. 2016, 53: 140-154.

[9]. Deming Lei. Co-evolutionary genetic algorithm for fuzzy flexible job shop scheduling [J]. Applied Soft Computing, 2012.

[10]. Jun-qing Li,Quan-ke Pan. Chemical-reaction optimization for flexible job-shop scheduling problems with maintenance activity [J]. Applied Soft Computing, 2012, 12: 2896-2912.

[11]. YU Sheng-ping, PANG Xin-fu, CHAI Tian you, et al. Research on production scheduling for steelmaking and continuous casting with processing time uncertainty [J]. Control and Decision, 2009, 24(10): 1467-1472. 